\title{
Re: Effect of Sacral Neuromodulation on Female Sexual Function and Quality of Life: Are They Correlated?
}

\author{
Mai Banakhar1, Yahya Gazwani1, Mohamed El Kelini1, Tariq Al-Shaiji2, Magdy Hassouna2 \\ 1 King Abdulaziz University Faculty of Medicine, Department of Urology, Jeddah, Saudi Arabia \\ 2 University of Toronto, Toronto Western Hospital, Department of Urology, Toronto, United States of America
}

Can Urol Assoc J 2014;8:e762-767.

\section{EDITORIAL COMMENT}

Sacral neuromodulation (SNM) has become an established therapy for voiding dysfunction (VD). There have been published papers that documented improvement in bowel functions and bladder pain via SNM. However, improvement in female sexual function (FSF) after SNM treatment remains unclear. Recently, Banakhar et al. evaluated the effects of SNM on FSF and its impact on quality of life (QoL) and analyzed any correlation. They evaluated 33 female patients who were treated with SNM for VD. All patients completed the Female Sexual Function Index (FSFI), the 36-Item Short-Form Health Survey (SF-36), and incontinence questionnaires (the Urinary Distress Inventory [UDI-6]) preoperatively and 4 months postoperatively. Ten patients were excluded from the study because they were not sexually active. The indications were: refractory overactive bladder in 19, frequency urgency syndrome in 2, and non-obstructive urinary retention in another 2 patients. SNM therapy significantly improved the total FSFI score ( $p=0.011)$. When the SF-36 and UDI-6 scores were compared, QoL showed significant improvement after SNM treatment. However, improvement in FSFI scores was not correlated with improvement in OoL. SNM therapy is an effective treatment modality in selected patient groups. In our country, SNM therapy has become a new option in the treatment of lower urinary tract problems. According to the results of this study, it is too early to conclude that SNM certainly improve FSF and QoL. The study just evaluated 23 cases. In order to have a more discrete conclusion, we need prospective trials with larger series.

\section{Re: The Value of Urodynamic Tools to Guide Patient Selection in Sacral Neuromodulation}

\author{
Jamie Drossaerts, Kevin Rademakers, Gommert van Koeveringe, Philip Van Kerrebroeck
}

Maastricht University Medical Centre (MUMC+), The Netherlands and School of Mental Health and Neuroscience (MHeNS), Maastricht

University, Department of Urology, Maastricht, Netherlands

World J Urol Doi: 10.1007/s00345-015-1479-6.

\section{EDITORIAL COMMENT}

Sacral neuromodulation (SNM) is recommended as a treatment option if conservative treatments either fail or lead to adverse events in patients with overactive bladder syndrome or non-obstructive urinary retention. Treatment success of these storage and voiding dysfunctions is evaluated in terms of improvement in micturition diaries, subjective patient evaluation, quality of life scores and symptom score questionnaires. In addition, treatment success in bladder dysfunctions is also evaluated with conventional urodynamic studies (UDS) and ambulatory UDS. This year, Drossaerts et al. have investigated 98 patients with lower urinary tract symptoms who underwent ambulatory UDS. All patients were already treated with SNM therapy. Success rate of SNM in patients with storage dysfunction was 70\%, according to either conventional UDS or ambulatory UDS diagnosis. Based on conventional UDS, success rate of SNM in patients with hypocontractility was $67 \%$, and in acontractile patients, 35\%. According to ambulatory UDS diagnosis, success rates were $32 \%$ and $17 \%$, respectively. The authors concluded that, in patients with storage dysfunction, conventional UDS predict the SNM treatment success as good as ambulatory UDS. However, conventional UDS overestimates the amount of patients diagnosed with hypocontractile or acontractile bladder. Thus, conventional UDS do not predict treatment success failure in those cases.

In my opinion, as the authors emphasized in the paper, the recording of a detrusor contraction can easily be detected via ambulatory UDS, although it is not validated yet. An acontractile or hypocontractile bladder confirmed on ambulatory UDS is therefore definitely more reliable to be a real acontractile/hypocontractile bladder. For this reason, ambulatory UDS rather than conventional UDS should be preferred in the diagnosis of acontractile bladder. By this way, detrusor contractility dynamics might better be identified. In the next step, when we diagnosed acontractile/hypocontractile bladders via ambulatory UDS, we should not prefer SNM therapy as a treatment option. Because, as we see in the current study, success rates of $32 \%$ and $17 \%$ show treatment failure. 